\title{
Imagem corporal, humor e qualidade de vida
}

\author{
Body image, mood and quality of life
}

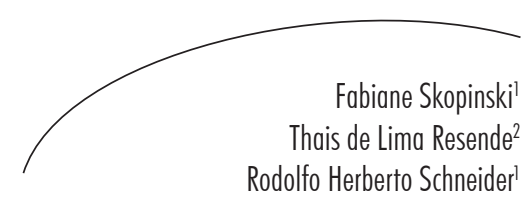

\section{Resumo}

Objetivo: Caracterizar, quanto a imagem corporal, humor e qualidade de vida, mulheres pós-menopáusicas que buscam atendimento de fisioterapia dermatofuncional, dermatologia e medicina estética. Materiais e Métodos: Estudo transversal com 46 mulheres que buscaram atendimento estético em três locais na cidade de Porto AlegreRS, Brasil. Foram coletados dados sociodemográficos e de saúde. Foram aplicados os instrumentos: Escala de Depressão Geriátrica (GDS-15), questionário de qualidade de vida WHOQOL-bref e Escala de Silhuetas de Stunkard. Para avaliar a associação entre as variáveis, os coeficientes de correlação de Pearson ou Spearman foram utilizados. O nível de significância adotado foi de $5 \%(\mathrm{p} \leq 0,05)$. As análises foram realizadas no programa SPSS versão 18.0. Resultados: Verificou-se que a maioria das participantes estava insatisfeita com sua imagem corporal, tinha alto nível de satisfação com sua qualidade de vida e não apresentava sintomatologia depressiva. Conclusão: Para as participantes do estudo, a satisfação com a imagem corporal está relacionada a melhor qualidade de vida, menor probabilidade de apresentar sintomas depressivos, menor índice de massa corporal e maior renda pessoal e familiar.

\section{Abstract}

Objective: To characterize body image, mood and quality of life in postmenopausal women who seek dermatological physiotherapy, dermatology and aesthetic medicine care. Materials and Methods: Cross-sectional study with 46 women who sought aesthetic care in three locations in the city of Porto Alegre-RS, Brazil. Sociodemographic and health data were collected. The following tools were used: Geriatric Depression Scale (GDS-15), Quality of life questionnaire WHOQOL-bref and Scale of Stunkard silhouettes. In order to evaluate the association between the variables, the correlation coefficients Pearson or Spearman were used. The level of significance was $5 \%(\mathrm{p} \leq 0.05)$.

\section{Palavras-chave:}

Envelhecimento. Idoso. Imagem Corporal. Depressão. Qualidade de Vida.

Key words: Aging. Elderly. Body Image. Depression. Quality of Life.

\footnotetext{
Pontifícia Universidade Católica do Rio Grande do Sul. Programa de Pós-graduação em Gerontologia Biomédica, Instituto de Geriatria e Gerontologia. Porto Alegre, RS, Brasil.

2 Pontifícia Universidade Católica do Rio Grande do Sul. Faculdade de Enfermagem, Nutrição e Fisioterapia. Porto Alegre, RS, Brasil.
} 
Analyses were performed using SPSS version 18.0. Results: It was found that most participants are dissatisfied with their body image, have a high level of satisfaction with their quality of life and have no depressive symptoms. Conclusion: In this study the participants' satisfaction with body image is associated with better quality of life, less likely to have depressive symptoms, lower body mass index and increased personal and family income.

\section{INTRODUÇÃOO}

A menopausa é uma etapa integrante do processo de envelhecimento feminino, representando um marco importante na vida da mulher, pois está associada a diversas mudanças biológicas, psicológicas e sociais. ${ }^{1,2}$

O envelhecimento está associado a mudanças físicas e psicológicas. O peso corporal aumenta tanto nos homens quanto nas mulheres até o final da meia-idade, quando o peso das mulheres se estabiliza e o dos homens diminui. A água corporal total diminui e ocorre a diminuição da flexibilidade e força muscular. Além das mudanças físicas, mudanças relacionadas à saúde na composição corporal, também há implicações importantes. A redistribuição e aumento da gordura corporal e perda da massa muscular resultam em diminuição na função aeróbia e aumento da gordura corporal, principalmente a abdominal, que está associada a outras doenças, além de influenciar a imagem corporal. ${ }^{3,4}$

O conceito de imagem corporal é a maneira pela qual a aparência física aparece para o indivíduo, correspondendo à representação mental do próprio corpo. ${ }^{5}$ As mudanças físicas que ocorrem com o envelhecimento, de maneira gradual, trazem para o indivíduo uma modificação de sua própria imagem e, muitas vezes, podem ocasionar uma diferença entre a imagem desejada e a imagem real. A forma como as pessoas percebem sua imagem corporal tem consequências importantes sobre sua saúde e qualidade de vida. As mulheres, ao longo do ciclo vital, possivelmente influenciadas por fatores culturais, manifestam mais interesse, preocupação e insatisfação com a imagem corporal em relação aos homens. ${ }^{6}$

Muitos fatores socioculturais, incluindo a mídia, podem afetar o padrão das pessoas com relação a sua imagem corporal. A autopercepção do peso corporal é um aspecto importante, visto que pode repercutir na visão que o idoso tem em relação ao seu corpo., ${ }^{2,7,8}$

Diante desta temática, considerando as mudanças que ocorrem no período que envolve a menopausa, o objetivo deste estudo foi caracterizar as mulheres pós-menopáusicas que buscam atendimento de fisioterapia dermatofuncional, dermatologia e medicina estética, quanto a imagem corporal, humor e qualidade de vida.

\section{MÉTODOS}

Estudo transversal desenvolvido no período de março a dezembro de 2013. A população-alvo foi composta por mulheres no período da pósmenopausa atendidas no serviço de Fisioterapia Dermatofuncional do Centro de Reabilitação do Hospital São Lucas da PUCRS, no Centro de Dermatologia do Centro Clínico da PUCRS e Consultório de Medicina Estética, todos no município de Porto Alegre-RS, Brasil. Foram incluídas mulheres no período da pós-menopausa que estivessem em atendimento junto aos serviços citados e excluídas as mulheres que se recusaram a responder os instrumentos de pesquisa.

As participantes responderam a um questionário desenvolvido pela pesquisadora, no qual foram coletados dados sociodemográficos e 
de saúde, incluindo idade; estado civil; ocupação; renda pessoal e familiar expressas em salário mínimo nacional (SM); escolaridade, estratificada em baixa (até o primeiro grau incompleto), média (primeiro grau completo até o ensino médio completo) e alta (ensino superior incompleto ou completo); o tempo de menopausa; o índice de massa corporal (IMC), além da Escala de Depressão Geriátrica (GDS-15), WHOQOLbref e Escala de Silhuetas de Stunkard.

A Escala de Depressão Geriátrica (GDS-15), que é uma versão reduzida da escala original elaborada por Sheikh \& Yesavage, ${ }^{9,10}$ validada no Brasil e amplamente utilizada para detecção de sintomas depressivos em pacientes idosos, foi utilizada para a determinação da presença ou não de sintomatologia depressiva. Composta por 15 perguntas negativas/afirmativas, um escore total igual a 5 ou mais pontos indica a presença de sintomas depressivos, sendo que um escore igual ou superior a 11 caracteriza depressão grave. $\mathrm{O}$ ponto de corte usado apresenta sensibilidade de $81 \%$ e especificidade de $71 \%$. Assim, um indivíduo cujo escore é igual ou superior a cinco pontos apresenta sintomas sugestivos de depressão, e com escore inferior a cinco pontos não apresenta sintomas sugestivos de depressão., ${ }^{9,10}$

A qualidade de vida foi mensurada por meio do questionário WHOQOL-bref, ${ }^{11-14}$ que é composto por 26 questões, com duas questões gerais e as demais 24 representando cada uma das 24 facetas que integram o instrumento original completo do qual foi derivado, o WHOQOL-100, composto por 100 questões. O WHOQOL-bref é composto por quatro domínios: Físico, Psicológico, Relações Sociais e Meio Ambiente, que avaliam, respectivamente: capacidade física, bem-estar psicológico, relações sociais e contexto ambiental. Cada domínio é composto por questões cujas alternativas variam de uma intensidade que vai de 1 a 5 . Em cada domínio é gerado um escore total de 100 pontos, sendo que, quanto mais alto o escore, mais bem classificada é a qualidade de vida. ${ }^{15}$
A satisfação com a imagem corporal foi determinada pela Escala de Silhuetas proposta por Stunkard et al., ${ }^{16,17}$ que consiste de desenhos com diferentes formas humanas, numerados de 1 a 9, sendo a primeira silhueta a mais magra e a nona a mais obesa. O conjunto de silhuetas foi apresentado às mulheres, que escolheram duas delas, a que melhor representava a sua aparência física atual e aquela que gostariam de ter. A pontuação da escala é dada pelo resultado da diferença do número obtido entre a silhueta corporal atual e a silhueta desejada. Assim, um sujeito cujo escore é igual a zero é considerado satisfeito com a sua imagem corporal, enquanto que qualquer outro escore indica insatisfação com a imagem corporal. ${ }^{16,17}$

As variáveis quantitativas foram descritas por média e desvio-padrão ou mediana e amplitude interquartílica. As variáveis categóricas foram descritas por frequências absolutas e relativas. Para avaliar a relação entre variáveis categóricas, foram utilizados o teste exato de Fisher e teste $t$ Student. Para avaliar a associação entre as variáveis, os coeficientes de correlação de Pearson ou Spearman foram utilizados. O nível de significância adotado foi de $5 \%(\mathrm{p} \leq 0,05)$. As análises foram realizadas no programa SPSS versão 18.0. O cálculo do tamanho amostral foi realizado levando em consideração as prevalências encontradas no presente estudo para os sintomas sugestivos de depressão e a satisfação com a imagem corporal. Como o tamanho amostral, baseado na prevalência de mulheres satisfeitas com a sua imagem corporal foi menor $(n=34)$, adotouse o tamanho amostral relativo aos sintomas sugestivos de depressão. Assim, assumindo um erro máximo admissível de $13 \%$, bem como um nível de significância de $5 \%(\alpha=0,05)$ e um poder de $80 \%$ ( $\beta=0,20)$, o tamanho mínimo de amostra foi estimado em 43 casos.

O presente estudo seguiu os princípios éticos da Declaração de Helsinki, sendo aprovado pelo Comitê de Ética em Pesquisa da Pontifícia Universidade Católica do Rio Grande do Sul (CEP: PB 191.315/2013). Todas as participantes 
assinaram o Termo de Consentimento Livre e Esclarecido.

\section{RESULTADOS}

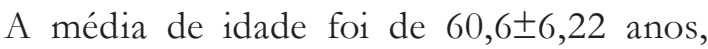
sendo a mais jovem com 49,3 anos e a mais idosa, 73,5 anos. O tempo de menopausa variou de 5,4 a 13,7 anos. A maior parte das participantes apresentou escolaridade de nível superior (69,6\%), era profissionalmente ativa $(54,3 \%)$ e tinha renda familiar superior a seis salários mínimos (50\%). Os dados gerais da amostra estão representados na tabela 1 .

Tabela 1. Caracterização socioeconômica da amostra. Porto Alegre-RS, 2014.

\begin{tabular}{|c|c|}
\hline Variáveis & $\mathrm{n}=46$ \\
\hline \multicolumn{2}{|l|}{ Idade (anos) } \\
\hline Média $\pm \mathrm{dp}$ & $60,6 \pm 6,22$ \\
\hline md (P25-P75) & $60,5(56,4-64,9)$ \\
\hline Idade mínima - máxima (anos) & $49,3-75,3$ \\
\hline Tempo de menopausa (anos) - md (P25 - P75) & $9,9(5,4-13,7)$ \\
\hline \multicolumn{2}{|l|}{ Escolaridade - n $(\%)$} \\
\hline Analfabeto & $1(2,2)$ \\
\hline $1^{\circ}$ grau & $3(6,5)$ \\
\hline $2^{\circ}$ grau & $7(15,2)$ \\
\hline Superior incompleto & $3(6,5)$ \\
\hline Superior completo & $32(69,6)$ \\
\hline \multicolumn{2}{|l|}{ Profissionalmente ativas $-\mathrm{n}(\%)$} \\
\hline Sim & $25(54,3)$ \\
\hline Não & $21(45,7)$ \\
\hline \multicolumn{2}{|l|}{ Renda pessoal - n (\%) } \\
\hline$<3 \mathrm{SM}$ & $7(15,2)$ \\
\hline 3 a 4 SM & $13(28,3)$ \\
\hline 4 a $6 \mathrm{SM}$ & $10(21,7)$ \\
\hline$>6 \mathrm{SM}$ & $13(28,3)$ \\
\hline NR & $3(6,5)$ \\
\hline \multicolumn{2}{|l|}{ Renda familiar - n $(\%)$} \\
\hline$<3 \mathrm{SM}$ & $3(6,5)$ \\
\hline 3 a 4 SM & $8(17,4)$ \\
\hline 4 a $6 \mathrm{SM}$ & $9(19,6)$ \\
\hline$>6 \mathrm{SM}$ & $23(50,0)$ \\
\hline NR & $3(6,5)$ \\
\hline \multicolumn{2}{|l|}{ Estado civil - n (\%) } \\
\hline Casado & $22(47,8)$ \\
\hline Separado/divorciado & $13(28,3)$ \\
\hline Viúvo & $7(15,2)$ \\
\hline Solteiro & $4(8,7)$ \\
\hline
\end{tabular}

$\mathrm{dp}=$ desvio-padrão; $\mathrm{md}$ (P25-P75)= mediana (intervalo interquartil); SM= salário mínimo; $\mathrm{NR}=$ não quiseram responder. 
Em relação ao IMC, 82,6\% das participantes apresentaram peso normal ou sobrepeso, e 54,3 $\%$ declararam não ser sedentárias, $91,3 \%$ negaram tabagismo e 54,3\% referiram consumir algum tipo de bebida alcoólica semanalmente. Quanto à satisfação em relação à imagem corporal e presença de sintomatologia depressiva, 84,8\% da amostra demonstraram insatisfação com a imagem corporal e 26,1\% apresentaram sintomas sugestivos de depressão (tabela 2).

Tabela 2. Caracterização da amostra em relação a dados antropométricos, estilo de vida, sintomatologia depressiva, imagem corporal e qualidade de vida. Porto Alegre-RS, 2014.

\begin{tabular}{lc}
\hline Variáveis & $\mathrm{n}=46$ \\
\hline IMC (kg/m2) - (n \%) & $19(41,3)$ \\
$\quad$ Normal (IMC 18,5 a $25 \mathrm{~kg} / \mathrm{m} 2)$ & $19(41,3)$ \\
$\quad$ Sobrepeso (IMC $25 \mathrm{a} 30 \mathrm{Kg} / \mathrm{m} 2)$ & $8(17,4)$ \\
$\quad$ Obesidade (IMC $\geq 30 \mathrm{~kg} / \mathrm{m} 2)$ & \\
Sedentarismo - n (\%) & $21(45,7)$ \\
$\quad$ Sim & $25(54,3)$ \\
$\quad$ Não & \\
Tabagista - n (\%) & $4(8,7)$ \\
Sim & $42(91,3)$ \\
$\quad$ Não & \\
Ingestão de bebida alcoólica - n (\%) & $25(54,3)$ \\
Sim & $21(45,7)$ \\
$\quad$ Não & \\
Imagem corporal - n (\%) & $7(15,2)$ \\
$\quad$ Satisfeitas & $39(84,8)$ \\
Insatisfeitas & \\
GDS-15 & $2(0,8-5)$ \\
Escore - md (P25-P75) & $12(26,1)$ \\
Com sintomas depressivos ( $\geq 5$ pontos) & $34(73,9)$ \\
Sem sintomas depressivos (<5 pontos) & \\
Qualidade de vida (WHOQOL-bref) - média \pm dp & $75,3 \pm 16,0$ \\
Domínio Físico & $73,5 \pm 14,1$ \\
Domínio Psicológico & $72,1 \pm 19,3$ \\
Domínio Social & $74,1 \pm 14,0$ \\
Domínio Ambiente & $75,5 \pm 17,9$ \\
$\quad$ Geral & \\
\hline
\end{tabular}


Não foram detectadas diferenças significativas entre as mulheres satisfeitas e insatisfeitas com sua imagem corporal em relação à presença de sintomas depressivos e qualidade de vida. Entretanto, nenhuma das mulheres satisfeitas com sua imagem corporal apresentou sintomas sugestivos de depressão, enquanto que 30,8\% das insatisfeitas com a imagem corporal o apresentaram. No que se refere à qualidade de vida, as mulheres satisfeitas apresentaram escores mais altos nos quatro domínios do WHOQOLbref (tabela 3).

Tabela 3. Presença de sintomas depressivos e qualidade de vida da amostra, de acordo com a satisfação/ insatisfação com a imagem corporal. Porto Alegre-RS, 2014.

\begin{tabular}{lccc}
\hline Variáveis & $\begin{array}{c}\text { Satisfeitas } \\
(\mathrm{n}=7)\end{array}$ & $\begin{array}{c}\text { Insatisfeitas } \\
(\mathrm{n}=39)\end{array}$ & $\mathrm{p}$ \\
\hline $\begin{array}{l}\text { Presença de sintomas depressivos* } \\
\text { Sim }\end{array}$ & $0(0,0)$ & $12(30,8)$ & $0,165^{\#}$ \\
$\quad$ Não & $27(69,2)$ & \\
Qualidade de vida** - média \pm dp & & & \\
$\quad$ Domínio Físico & $85,2 \pm 9,8$ & $73,5 \pm 16,4$ & $0,076^{\# \#}$ \\
Domínio Psicológico & $81,5 \pm 9,6$ & $72,0 \pm 14,4$ & $0,101^{\# \#}$ \\
Domínio Social & $76,2 \pm 17,6$ & $71,4 \pm 19,7$ & $0,548^{\# \#}$ \\
Domínio Ambiente & $76,8 \pm 13,4$ & $73,6 \pm 14,3$ & $0,590^{\# \#}$ \\
Geral & $75,0 \pm 23,9$ & $75,6 \pm 17,0$ & $0,932^{\# \#}$
\end{tabular}

*Escala de depressão geriátrica (GDS-15); ${ }^{* * W H O Q O L-b r e f ; ~ d p=~ d e s v i o-p a d r a ̃ o ; ~}{ }^{\#}$ teste exato de Fisher; ${ }^{\# \#}$ teste $t$ Student.

A relação entre o escore da escala de depressão geriátrica e a diferença no escore de imagem corporal mostrou associação positiva entre os escores dos dois instrumentos $(\mathrm{r}=0,367 ; \mathrm{p}=0,012)$, ou seja, as mulheres mais insatisfeitas com a imagem corporal apresentaram escores mais elevados de sintomas sugestivos de depressão.

Também foi investigada a relação entre a insatisfação com a imagem corporal e a qualidade de vida, na qual foi detectada associação inversa significativa nos domínios "físico", "psicológico" e "ambiente". Ou seja, quanto maior a insatisfação com a imagem corporal, pior é a qualidade de vida percebida nesses domínios (tabela 4). Em relação ao escore de sintomatologia depressiva, a associação inversa foi significativa com todos os domínios de qualidade de vida, conforme mostra a tabela 4. 
Tabela 4. Associação do escore de depressão e imagem corporal com qualidade de vida. Porto AlegreRS, 2014.

\begin{tabular}{lccccc}
\hline Variáveis & \multicolumn{4}{c}{ Escores do WHOQOL-bref } \\
& Físico & Psicológico & Social & Ambiente & Geral \\
& $\mathrm{r}$ & $\mathrm{r}$ & $\mathrm{r}$ & $\mathrm{r}$ & $\mathrm{r}$ \\
\hline Imagem corporal & $-0,393^{* *}$ & $-0,355^{*}$ & $-0,123$ & $-0,329^{*}$ & $-0,240$ \\
Escore de depressão & $-0,519^{* * *}$ & $-0,678^{* * *}$ & $-0,374^{*}$ & $-0,444^{* *}$ & $-0,470^{* *}$ \\
\hline
\end{tabular}

$\mathrm{r}=$ coeficiente de correlação de Spearman; ${ }^{\mathrm{p}}<0,05 ; * * \mathrm{p}<0,01 ; * * * \mathrm{p}<0,001$.

As variáveis IMC, tempo de menopausa, renda pessoal, renda familiar, escolaridade e idade com os desfechos insatisfação com a imagem corporal e escore de sintomatologia depressiva, mostraram que o IMC apresentou correlação positiva com a imagem corporal - ou seja, quanto maior o IMC, maior é a insatisfação com a imagem corporal. As variáveis "renda pessoal" e "escolaridade" apresentaram correlação negativa com a imagem corporal, ou seja, quanto maior a renda pessoal, familiar e escolaridade, menor é a insatisfação com a imagem corporal. Da mesma forma, houve correlação negativa da renda familiar com o escore de sintomas depressivos, onde quanto maior a renda familiar, menor é a presença de sintomatologia depressiva. Essas associações podem ser visualizadas na tabela 5 .

Tabela 5. Associação de diferentes variáveis e os desfechos "imagem corporal" e "escore de sintomas depressivos”. Porto Alegre-RS, 2014.

\begin{tabular}{lcc}
\hline Variáveis & Imagem corporal & Escore de sintomas depressivos \\
\hline IMC & $\mathrm{r}$ & $\mathrm{r}$ \\
Tempo de menopausa & $0,522^{* * *}$ & 0,139 \\
Renda pessoal & 0,135 & $-0,059$ \\
Renda familiar & $-0,432^{* *}$ & $-0,247$ \\
Nível de escolaridade & $-0,361^{*}$ & $-0,302^{*}$ \\
Idade & $-0,348^{*}$ & $-0,084$ \\
\hline
\end{tabular}

$\mathrm{IMC}=$ índice de massa corporal; $\mathrm{r}=$ coeficiente de correlação de Spearman; ${ }^{*} \mathrm{p}<0,05$;

${ }^{*}{ }^{\mathrm{p}}<0,01 ; * * * \mathrm{p}<0,001$ 


\section{DISCUSSÃO}

$\mathrm{O}$ presente estudo mostrou que, embora o grupo participante fosse composto por um grupo de idosas jovens ${ }^{18}$ casadas, com escolaridade mais elevada, profissionalmente ativas e com renda familiar superior a seis salários mínimos, a maior parte demonstrou estar insatisfeita com sua imagem corporal e não apresentou sintomas sugestivos de depressão. Essa situação parece estar relacionada com as mudanças corporais e estéticas que o envelhecimento traz consigo. Não obstante, na literatura, em sua maioria, os assuntos mais abordados são relativos às alterações orgânicas da senescência, como diabetes mellitus, hipertensão arterial sistêmica, obesidade, dislipidemia e osteoporose, incontinência urinária, ${ }^{3,16}$ além da presença de depressão e índices de qualidade de vida. ${ }^{19-}$ 22 Estudos que avaliem os aspectos estéticos relacionados ao envelhecimento ainda são incipientes. Os estudos atuais, em sua maioria, são referentes a procedimentos cirúrgicos ou mais direcionados aos aspectos morfofuncionais do envelhecimento cutâneo.

A insatisfação das participantes do estudo com sua imagem corporal, bem como sua associação com a presença de sintomas depressivos, condiz com os achados de Sarabia Cobo, ${ }^{6}$ que avaliou 198 idosos com o Body Shape Questionnarire e a Escala de Silhuetas de Stunkard. Desenvolvido em Santander, na Espanha, demonstrou que a maior parte da amostra feminina estava insatisfeita com sua imagem corporal e apresentava depressão ou diagnóstico de depressão prévia. ${ }^{6}$

Outro desfecho importante encontrado na presente pesquisa em relação à qualidade de vida apresentou relação com a imagem corporal em três dos quatro domínios ("físico", "psicológico" e "ambiente"), de tal forma que as mulheres satisfeitas também tinham melhor qualidade de vida nesses domínios. É possível que a forma como os indivíduos percebem sua imagem corporal tenha impacto na sua qualidade de vida de tal forma que uma imagem positiva sobre si reflete uma adaptação e/ou aceitação das mudanças físicas relacionadas ao envelhecimento, enquanto que uma atitude negativa reforça os sintomas depressivos, a baixa autoestima e falta de motivação. ${ }^{6}$ Estudos têm demonstrado que a forma percebida pela mulher em relação às mudanças físicas relacionadas ao período da pós-menopausa, impacta diretamente na sua qualidade de vida. ${ }^{8}$

Talvez fosse esperada uma diferença significativa na comparação entre as mulheres satisfeitas e insatisfeitas com sua imagem corporal em relação à prevalência de sintomas sugestivos de depressão e melhor qualidade de vida, o que não aconteceu. Não obstante, pode ser visto que, enquanto um terço das mulheres insatisfeitas com sua imagem corporal apresentou sintomas sugestivos de depressão, nenhuma apresentou esses sintomas entre as satisfeitas. Pode-se observar que, em todos os domínios, os escores das mulheres satisfeitas são mais elevados, com as diferenças variando entre $4 \%$ no domínio "ambiente" e 15\% no "físico".

Em relação à escolaridade, Trentini ${ }^{23}$ verificou que esta variável teve associação forte com a intensidade de depressão. A autora ${ }^{23}$ mostrou que essa associação ocorre de forma inversa, ou seja, quanto maior a escolaridade, menor a intensidade de sintomas depressivos ou de somatizações. Desta forma, quanto maior o nível de escolaridade, maior seria o fator de proteção para sintomas depressivos. É possível que a alta escolaridade contribua para melhor compreensão das mudanças corporais, reduza a ansiedade e estimule o autocuidado. ${ }^{2}$

Segundo Coelho \& Fagundes, ${ }^{24}$ existe relação entre satisfação com a imagem corporal e renda. Esses autores mostraram que as mulheres de maior poder aquisitivo se perceberam relativamente magras, mas almejavam continuar perder peso, enquanto que as de renda familiar menor perceberam-se relativamente obesas, mais distantes do modelo de corpo ideal, mas desejavam uma silhueta menor. ${ }^{24}$ Os últimos dados corroboram o presente estudo, segundo o qual mulheres com renda pessoal e familiar maior estão mais satisfeitas com a imagem corporal quando comparadas às de menor renda, mas ainda assim buscam melhorar sua aparência. 
Coelho \& Fagundes ${ }^{24}$ sugerem que o modelo de beleza imposto socialmente afeta as mulheres, independentemente da classe econômica.

Confirmando a associação inversa entre sintomas sugestivos de depressão e qualidade de vida, ${ }^{14,23}$ as mulheres do presente estudo com indícios de depressão apresentaram pior qualidade de vida em todos os domínios investigados. Ao que parece, indivíduos que avaliam sua qualidade de vida como negativa apresentam significativamente mais sintomas depressivos do que aqueles com esperança e que relatam melhores condições de saúde. ${ }^{14}$ Não se pode ignorar que as mulheres estudadas estão no período da pós-menopausa, o qual é influenciado por mudanças hormonais que podem levar a alterações de humor, diminuição da libido, distúrbios do sono, fogachos, aumento da gordura corporal, o que pode repercutir na sua vida social e profissional. . $^{1,2,8,25}$

Ainda em relação aos sintomas depressivos relacionados à pós-menopausa, é possível que esses sintomas estejam relacionados a dificuldades emocionais prévias, o que pode comprometer a percepção das mulheres acerca dessa fase da vida. ${ }^{2}$ Outro fator importante a ser considerado é a maior vulnerabilidade das mulheres à perda da autoestima em relação aos homens, ${ }^{26}$ que nessa fase da vida é acentuada pelo aparecimento das rugas, a perda do corpo jovem e o medo de envelhecer, podendo afetar negativamente a qualidade de vida. ${ }^{26}$

As relações entre a renda familiar e a presença de sintomas sugestivos de depressão, bem como os escores dos domínios "ambiente" e "social" do WHOQOL-bref indicam que as mulheres com melhor condição financeira têm melhor qualidade de vida nesses domínios e são menos sujeitas à depressão. Esses achados são corroborados por aqueles relatados por Pereira, Alvarez \& Traebert $^{27} \mathrm{em}$ idosos. Os autores ainda detectaram associação entre baixa renda e baixa escolaridade com pior percepção de qualidade de vida no domínio "ambiente". 15,27 Ao considerar que este domínio aborda questões relacionadas a segurança física, disponibilidade de recursos financeiros, oportunidades de adquirir novas informações, recreação e lazer e disponibilidade de cuidados com a saúde,15 a associação poderia ser explicada pelo fato de tais variáveis demonstrarem maior exposição da pessoa idosa a piores condições de moradia e segurança física. Dessa forma, oportunidades novas de informações e acesso aos serviços de saúde poderiam ser diminuídas.

Sabe-se que tanto a escolaridade quanto o nível econômico são importantes marcadores socioeconômicos. Estudos relatam que a pobreza pode ser determinante na explicação do aumento dos índices de depressão, pois estes estão associados a condições sociais como desemprego, baixo nível de instrução, baixa qualidade de moradia e alimentação inadequada..$^{28,29}$

O presente estudo mostrou que mulheres com maior renda e nível de escolaridade também apresentaram maior satisfação com a imagem corporal e isto pode estar relacionado a um melhor entendimento sobre as mudanças corporais que ocorrem, sobretudo no período da menopausa, estando essas mulheres em melhores condições de acesso aos tratamentos de pele, estéticos, de saúde e bem-estar.

As mudanças relacionadas ao envelhecimento fazem com que a população, principalmente a feminina, busque serviços e intervenções estéticas com a intenção de melhorar sua aparência física, ${ }^{30}$ ou seja, atenuar as mudanças externas que a menopausa e/ou o envelhecimento podem ocasionar. Assim sendo, é provável que as mudanças decorrentes do envelhecimento influenciem negativamente a satisfação com a imagem corporal, humor e qualidade de vida das mulheres.

É importante considerar que as mulheres avaliadas neste estudo são usuárias de serviços de estética e, desta forma, motivadas e preocupadas com aspectos relacionados à sua imagem corporal, independentemente do estado do humor, podendo este aspecto representar uma limitação do estudo. Este fato pode não espelhar necessariamente a realidade da população feminina que está envelhecendo e vive na comunidade, talvez por existir nesse grupo 
populacional uma preocupação mais direcionada às doenças crônicas, deixando a atenção com a imagem corporal em segundo plano.

\section{CONCLUSÃO}

O presente estudo mostrou que, em sua maioria, as mulheres pós-menopáusicas que buscam atendimento de fisioterapia dermatofuncional, dermatologia e medicina estética estão insatisfeitas com sua imagem corporal, porém têm alto nível de satisfação

\section{REFERÊNCIAS}

1. Poli MEH, Schwanke CHA, Da Cruz IBM. A Menopausa na Visão Gerontológica. Sci Med 2010;20(2):176-84

2. De Lorenzi DRS, Baracat EC, Padilha I Jr. Fatores associados à qualidade de vida após a menopausa. Rev Assoc Med Bras 2006; 52(5):312-17.

3. Spirduso WW. Dimensões Físicas do Envelhecimento. Barueri: Manole; 2005.

4. Damasceno VO, Vianna VRA, Vianna JM, Lacio M, Lima JRP, Novaes JS. Imagem corporal e corpo ideal. Rev Bras Ciênc Mov 2006;14(1):87-96.

5. Tavares MCGCF. Imagem corporal: conceito e desenvolvimento. Barueri: Manole; 2003.

6. 6.Sarabia Cobo CM. La imagen corporal em los ancianos. GEROKOMOS 2012;23(1):15-8.

7. Conte EMT. Indicadores de qualidade de vida em mulheres idosas. Rev Bras Cineantropom Desempenho Hum 2005;7(2):111-8.

8. De Lorenzi DRS, Catan LB, Cusin T, Felini R, Bassani F, Arpini AC. Caracterização da qualidade de vida segundo o estado menopausal entre mulheres da Região Sul do Brasil. Rev Bras Sáude Matern Infant 2009;9(4):459-466.

9. Almeida OP, Almeida AS. Confiabilidade da versão brasileira da escala de depressão em geriatria (GDS) versão reduzida. Arq Neuropsiquiatr 1999;57(2B):421-6.

10. Paradela EMP, Lourenço RA, Veras RP. Validação da escala de depressão geriátrica em um ambulatório geral. Rev Saúde Pública 2005; 39(6):918-23. com sua qualidade de vida e não apresentam sintomatologia sugestiva de depressão. Nesse grupo, a satisfação com a imagem corporal está relacionada a melhor qualidade de vida, menor probabilidade de apresentar sintomatologia sugestiva de depressão, menor índice de massa corporal e maior renda pessoal e familiar.

Futuros estudos são necessários para estabelecer novas conexões no complexo entendimento que envolve envelhecimento, mudanças estéticas e sua repercussão.

11. Organização Mundial da Saúde. WHOQOL abreviado: versão em português. Porto Alegre: OMS; 1998 [acesso em 13maio 2012]. Disponível em: http:// www.ufrgs.br/psiq/whoqol84.html.

12. Paskulin LMG. Fatores associados à qualidade de vida dos idosos de um distrito sanitário de Porto Alegre/ RS [tese]. São Paulo: Universidade Federal de São Paulo; 2006 [acesso em XX jun 2012]. Disponível em: http://ged1.capes.gov.br/CapesProcessos/926865ARQ/926865_4.PDF.

13. Fraquelli AA. A relação entre auto-estima, autoimagem e qualidade de vida em idosos participantes de uma oficina de inclusão digital [dissertação]. Porto Alegre: Pontifícia Universidade Católica do Rio Grande do Sul; 2008.

14. Irigaray TQ, Schneider RH. Dimensões de personalidade, qualidade de vida e depressão em idosas. Psicol Estud 2008;25(4):517-25.

15. Fleck MP. O instrumento de avaliação de qualidade de vida da Organização Mundial de Saúde (WHOQOL-100): características e perspectivas. Ciênc Saúde Coletiva 2000;5(1):33-8.

16. Do Nascimento LMP, Amaral RM, De Menezes RL, Sandoval RA. Percepção da imagem corporal, autoestima e qualidade de vida em alunos da UNATI/ UCG. Rev Digit B Aires 2008;13(127):1-10.

17. Pereira EF, Teixeira CS, Borgatto AF, Daronco LSE. Relação entre diferentes indicadores antropométricos e a percepção da imagem corporal em idosas ativas. Rev Psiquiatr Clín 2009;36(2):54-9. 
18. Papalia DE, Olds SW, Feldman RD. Desenvolvimento Humano. Porto Alegre: Artmed; 2006.

19. Pinto JM, Neri AL. Fatores associados à baixa satisfação em residentes na comunidade de idosos: Estudo FIBRA. Cad Saúde Pública 2013;29(12):2447-58.

20. Sposito G, D'Elboux MJ, Neri AL, Guariento ME. A satisfação com a vida e funcionalidade entre pacientes idosos em ambulatório de geriatria. Ciênc Saúde Coletiva 2013;18(12):3475-82.

21. Campos IC, Carvalho HN, Fernandes L. [Envelhecimento, sintomas circadianos e transtornos depressivos: uma revisão]. Am J Neurodegener Dis 2013;2(4):228-46. Inglês.

22. Reynolds III CF, Cuijpers P, Patel V, Cohen A, Dias A, Chowdhary N, et al. [Intervenção precoce para reduzir a carga de saúde global e econômica da depressão maior em idosos]. Ann Rev Public Health 2012;33:123-35. Inglês.

23. Trentini CM. Qualidade de Vida em idosos [tese]. Porto Alegre: Universidade Federal do Rio Grande do Sul; 2004.
24. Coelho EJN, Fagundes TF. Imagem Corporal de mulheres de diferentes classes econômicas. Motriz Rev Educ Fís 2007;13(2 Supl):S37-43.

25. Garciá-Portilla MP. Depression and perimenopause: a review. Actas Esp Psiquiatr 2009;37(4):213-21.

26. Sadock BJ, Sadock VA. Compêndio de psiquiatria: ciências do comportamento e psiquiatria clínica. Porto Alegre: Artmed; 2010.

27. Pereira KCR, Alvarez MA, Traebert JL. Contribuição das condições sociodemográficas para a percepção da qualidade de vida em idosos. Rev Bras Geriatr Gerontol 2011;14(1):85-95.

28. World Health Organization. The world health report. Mental health: new understanding, new hope. Geneva: OMS; 2001.

29. Mello MF, Mello AAF, Kohn R. Epidemiologia da saúde mental no Brasil. Porto Alegre: Artmed; 2007.

30. Audino MCF, Schmitz A. Cirurgia plástica e envelhecimento. Rev Bras Ciênc Envelhec Hum 2012:9(1):21-6. 